To the Editors:

\title{
Prevalence of methicillin resistant Staphylococcus aureus (MRSA) bacteremia at Teaching Hospital Anuradhapura, Sri Lanka
}

\author{
A S Jayaweera ${ }^{1}$, M Karunarathne ${ }^{3}$, W W Kumbukgolla ${ }^{2}$, H L Thushari ${ }^{3}$ \\ Ceylon Medical Journal 2017 62: 110-111 \\ DOI: http://doi.org/10.4038/cmj.v62i2.8478
}

Worldwide, an estimated 2 billion healthy people carry Staphylococcus aureus (SA) and of these, up to 53 million are thought to carry Methicillin Resistant Staphylococcus aureus (MRSA). SA is a feared pathogen because it causes severe infections and spread by metastatic foci. SA commonly colonizes the nostrils, skin, perineum, respiratory tract, open wounds and urinary tract which serves potential sites for infection. The multidrug resistant strains of SA have been isolated. In general, the fatality rates of MRSA infections range from $20 \%$ to $50 \%[1]$.

This retrospective study was conducted in Teaching Hospital Anuradhapura (THA), Sri Lanka. Microbiological investigations were done using the BacTec ${ }^{\circledR}$ 3D automated blood culture machine and the disc diffusion test (CLSI) was performed to assess the antibacterial susceptibility. Cases of culture-proven MRSA bacteremia over 18-month period were enrolled in the study. Demographic and clinical data were obtained using bed head tickets and laboratory records. Approval for the study was obtained from Ethical Review Committee, Faculty of Medicine and Allied Sciences, Rajarata University of Sri Lanka.

During the 18-month period, 13,260 blood cultures were investigated for possible bacteremia and 1352 of those were identified as bacteremia and the presence of SA bacteremia was detected in 655 out of 1352 cultures. Of these 61 (9.3\%) indicated the presence of MRSA. The highest prevalence (52.4\%) of MRSA bacteremia was observed among the people who aged $>60$ years. Also, it was detected more commonly in males (67.3\%) than the females (32.7\%). Nephrology unit 15 (24.6\%), orthopedic unit 14 (23\%) and ICUs 13 (21.3\%) had comparatively higher number of MRSA bacteremia cases.
The average period of hospitalization of a patient with MRSA bacteremia was $26.2 \pm 6.4$ days.

When the risk factors for acquisition of MRSA bacteremia is considered 26 (40\%) had a peripheral cannula, 16 (42.6\%) were on peritoneal dialysis, 16 (26.2\%) were with a urinary catheter, 4 (6.5\%) had an A-V shunts and 6 (9.8\%) were intubated. In addition, 10 (16.4\%) had undergone previous surgeries and 5 (8.2\%) were on chemotherapy. Ten (16.4\%) had with poly trauma. Fever (80.8\%) and inflammation at the entry site of medical device or incision site (78.8\%) were the commonest clinical manifestations.

Results of our study show, 9.3\% with SA bacteremia had MRSA. Of the total cases of bacteremia $4.5 \%$ had MRSA. Prevalence of MRSA bacteremia in Europe varies widely ( $0 \%$ in Iceland to $52.4 \%$ in Malta). Some countries like France, United Kingdom and Slovenia have demonstrated a decline in MRSA bacteremia rates over the recent years due to the implementation of multifaceted prevention programs [2]. In South-East Asian countries, the mortality rate associated with serious $S$ aureus infection was high, as much as $50 \%$ [3]. There are only a few studies on MRSA bacteremia in Sri Lanka. Most of the studies have looked at MRSA skin and soft tissue infections and colonization rates in patients and health care professionals [4]. Compared to most countries, this study shows a shows a comparatively low prevalence of MRSA bacteremia.

Hospital acquired MRSA (HAMRSA) infection is defined by the Center for Disease Control, Atlanta, USA as those in which patients had hospitalization, surgery, dialysis, residence in a long-term care facility or use of indwelling catheters in the previous 12 months. We found that in Teaching Hospital Anuradhapura, HAMRSA bacteremia was 3.03\%. In 2003, a study conducted at a

Departments of ${ }^{1}$ Microbiology and ${ }^{2}$ Biochemistry, Faculty of Medicine and Allied Sciences, Rajarata University, Saliyapura, Sri Lanka, ${ }^{3}$ Department of Microbiology, Teaching Hospital, Anuradhapura, Sri Lanka.

Correspondence: ASJ, e-mail: <jaas820703@yahoo.com>. Received 23 January 2017 and revised version accepted 17 March 2017.

This is an open-access article distributed under the terms of the Creative Commons Attribution License, which permits unrestricted use, distribution, and reproduction in any medium, provided the original author and source are credited. 
male surgical unit, National Hospital of Sri Lanka in Colombo reported that the prevalence of HAMRSA bacteremia was $6 \%$ [5]. This could be due the fact that surgical units are prone to acquire MRSA.

The highest percentage (24.6\%) of MRSA bacteremia was found in the nephrology unit. This is probably due to the large number of dialysis patients resulting from CKDu epidemic which prevails in the North Central Province. Introduction of peritoneal dialysis catheters and arterio-venous lines can significantly increase the likelihood of acquiring MRSA bacteremia. Though these procedures were routinely done under sterile conditions, adherence to standard precautions and assessment of sterility need to be done periodically.

Vancomycin can be used to treat MRSA bacteremia effectively. However, vancomycin has a potential nephrotoxic effect and there is high prevalence of chronic kidney disease in the North Central Province. This fact can discourage the doctors from prescribing vancomycin against MRSA bacteremia in THA. A prospective study to assess clinical efficacy related to timely introduction of anti-MRSA medication and MRSA bacteremia severity is recommended.

\section{Acknowledgement}

We acknowledge the support of TL Tennakoon who entered data and maintained the database and LD Dissanayake who retrieved data from the record room.

\section{Conflicts of interest}

There are no conflicts of interest.

\section{References}

1. Cheng AFB, French GL. Methicillin-resistant Staphylococcus aureus bacteremia in Hong Kong. J Hosp Infect 1988: 1291-01.

2. Lentino JR, Hennein H, Krause S, et al. A comparison of pneumonia caused by gentamicin, methicillin-resistant and gentamicin, methicillin-sensitive Staphylococcus aureus: epidemiologic and clinical studies. Infect Control 1985; 6: 267-72.

3. Jayaweera JAAS, Karunarathne M, Kumbukgolla WW, et al. The importance of timely introduction of vancomycin therapy against methicillin-resistant Staphylococcus aureus (MRSA) bacteremia and severity of MRSA bacteremia at Teaching Hospital, Anuradhapura, Sri Lanka. IJOH. 2017; 7: 11.

4. Abeygoonawardena $\mathrm{H}$, Navarathne V, Balasuriya A. Prevalence of Methicillin Resistant Staphylococcus aureus and Minimum Inhibitory Concentration (MIC) of Vancomycin Against it in Two Selected Semi Urban Sri Lankan Communities. IJSR 2015; 4: 393-95.

5. Corea E, de Silva T, Perera J. Methicillin-resistant Staphylococcus aureus: prevalence, incidence and risk factors associated with colonization in Sri Lanka. J Hosp Infec 2003; 55: 145-8. 